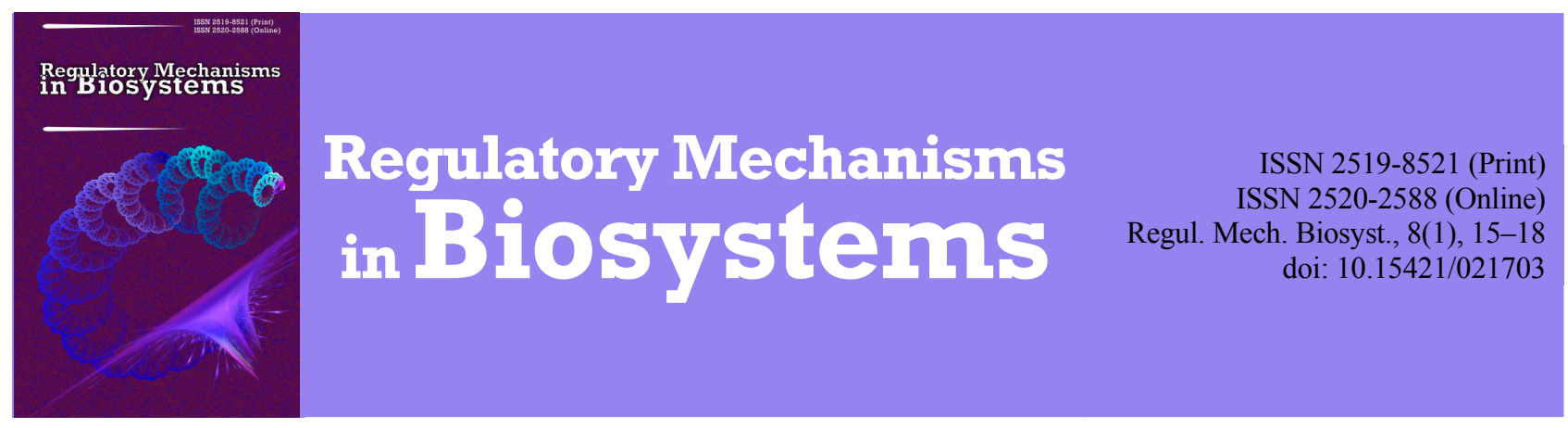

\title{
Reactions of eosinophilic granulocytes in the sputum and peripheral blood of children suffering from bronchial asthma with signs of eosinophilic and non-esinophilic inflammation of the bronchi
}

\author{
O. K. Koloskova, T. M. Bilous, M. V. Dikal, U. V. Lomakina, T. G. Kopchuk, T. O. Lobanova \\ Bukovinian State Medical University, Chernivtsi, Ukraine
}

Article info

Received 19.12.2016

Received in revised form 03.02.2017

Accepted 07.02.2017

Bukovinian State Medical University, Teatralna Square, 2 Chernivtsi, 58002, Ukrain Tel.: +38-050-221-35-16 E-mail:

koloskov-elena@yandex.ru. bilous.tetiana@bsmu.edu.ua vitmar888@mail.ru

\begin{abstract}
Koloskova, O. K., Bilous, T. M., Dikal, M. V., Lomakina, U. V., Kopchuk, T. G., \& Lobanova, T. O. (2017). Reactions of eosinophilic granulocytes in the sputum and peripheral blood of children suffering from bronchial asthma with signs of eosinophilic and non-eosinophilic inflammation of the bronchi. Regulatory Mechanisms in Biosystems, 8(1), 15-18. doi: 10.15421/021703
\end{abstract}

The aim of research was to improve the management of bronchial asthma in children by examining the peculiarities and diagnostic value of reaction markers of eosinophil granulocytes in the sputum and peripheral blood of patients with signs of eosinophil and non-eosinophil phenotypes of this disease. A cohort of 118 school-age children suffering from BA was examined during a period free from attacks. Group I (the main group ) included 61 schoolchildren with signs of eosinophil phenotype (EP) of asthma detected by the character of bronchial inflammation with eosinophil granulocytes present in the sputum at a level of $>3 \%$, group II (the comparison group) included 57 patients with a lower number of eosinophils in the sputum (non-eosinophil phenotype (NP) of BA). The average index of the relative content of eosinophils in the peripheral blood among the representatives of group I was $5.82 \pm 0.63 \%$, and in children with the signs of NPBA $-6.02 \pm 0.74 \%(\mathrm{P}>0.05)$, and average indices in the groups of absolute eosinophil number in the blood were $0.37 \pm 0.04$ and $0.41 \pm 0.05$ respectively $(\mathrm{P}>0.05)$. The negative reserve of NBT eosinophils in the sputum as a test to verify EPBA showed the following diagnostic values: specificity $-83.3 \%$, predicted value of a positive result 95.6\%. IL-5 content in the blood serum of children with EPBA was $5.99 \pm 1.74 \mathrm{ng} / \mathrm{ml}$, in patients of group II - only $1.99 \pm 0.49 \mathrm{ng} / \mathrm{ml}(\mathrm{P}<0.05)$. Eosinophil cationic protein $(\mathrm{ECP})$ in the sputum of patients of group I reached $2.72 \pm$ $0.35 \mathrm{ng} / \mathrm{ml}$, and in the comparison group - only $1.74 \pm 0.34 \mathrm{ng} / \mathrm{ml}(\mathrm{P}<0.05)$, when the content of ECP in sputum was $>1.0 \mathrm{ng} / \mathrm{ml}$ the risk of EPBA showed a statistically significant increase: $\mathrm{OR}=4.13, \mathrm{RR}=2.02$, and $\mathrm{AR}-0.34$. The efficacy of the standardized basic anti-inflammatory therapy in patients of clinical group I was higher as compared to the children with the signs of NPBA, which was illustrated by the reduced risk of inadequate control of the disease: the index of absolute risk decrease was $31.7 \%$, relative risk $-57.1 \%$ with necessary minimal number of patients -1.75 .

Keywords: NBT-test; airway inflammation; eosinophil cationic protein; asthma

\section{Introduction}

Nowadays bronchial asthma in children is considered as a disease characterized by chronic inflammation of the bronchi, in the development of which various cells and cellular elements play a certain role (Global Strategy for Asthma Management and Prevention, 2016). In case of antigenic stimulation, primary effector cells (epithelial cells of the respiratory tract, mast cells, macrophages) bound with $\mathrm{IgE}$ are considered to release mediators of inflammation resulting in the development of an inflammatory reaction both of an immediate and late type. The main mediators evaluating acute phase of inflammation are interleukins, tumor- $\alpha$ necrosis factor, interferon- $\gamma$, activating a range of immunological reactions and promoting the release of histamine, leukotrienes, eosinophil hemotaxis factors, neutrophils and other mediators of inflammation into the peripheral circulation (Chatila et al., 2008). Acidophilic leukocytes described more than 120 years ago by Paul Ehrlich and named as "eosinophils" were and still remain a matter of high scientific interest in different branches of medicine. Since that time asthma and helminthiasis and acute anaphylaxis have been associated with them and eosinophilia of the peripheral blood as a pathogenic syndrome of asthma was examined at the very beginning of the XX century. However, the role of these cells in occurrence of epithelial injury and other phenomena of late phase allergic inflammation was defined only during the last decade of the last century (Beasley et al., 1989). Eosinophils constitute from 1\% to $4 \%$ of the total number of leukocytes $\left(120-350\right.$ cells $\left./ \mathrm{mm}^{2}\right)$. They are of a specific granularity approximately 200 granules per cell. The granules contain alkaline proteins, the majority of them being large principal protein, eosinophil cationic protein, eosinophil peroxidase, and eosinophil neurotoxin (Motojima et al., 1989). Proinflammatory properties have been found in eosinophils due to those substances which are characterized by cytotoxicity and aid stimulation of mast cells and basophils degranulation, and therefore detection of the content of cationic proteins and peroxidase can be indicative of the degree of activation of the major proinflammatory cells of an allergic inflammation, and indirectly - of severity of this inflammatory process. At the same time, eosinophils are mainly tissue cells and this fact complicates the study of their functional peculiarities (Novitskiy et al., 2006).

In literary sources the bulk of attention has been paid to the detection of cytotoxic substances of eosinophil granulocytes in the 
peripheral blood and their relation to the severity of disorders in the general condition of patients suffering from allergic processes (Litvinova et al., 2006). Considering the leading role of eosinophils in allergic inflammation which occurs with bronchial asthma (Pavord, 2013), the study of the diagnostic and prognostic value of various indices of eosinophil granulocyte activity remains a high priority task for allergology (Jacobsen et al., 2014). At the same time, the highest informational value undoubtedly attaches to the above mentioned markers of acidophilic leukocytes activity located directly in the "shock" organ of bronchial asthma - in the respiratory tract. Literary data concerning the use of changes of eosinophil metabolic activity to improve the control of bronchial asthma (BA) in children are sparse and contradictory. Some of these sources contain sufficient diagnostic value of the given inflammatometric markers (Madison, 2000), while other researchers are convinced that the use of indices of eosinophil leukocyte activity in the blood alone is a test with insufficient information value to confirm the presence of BA in children (Kato et al., 2006). However, an increased number of eosinophils in the peripheral blood and respiratory tract, increased content of cationic proteins in the blood serum and shock-organs of patients with allergic pathology are considered by a number of authors as markers of unfavourable prognosis of bronchial-obstructive conditions in children (Jens et al., 2015).

This article aims at improving the management of bronchial asthma in children by examining the peculiarities and diagnostic value of reaction markers of eosinophil granulocytes in the sputum and peripheral blood of patients with signs of eosinophil and noneosinophil phenotypes of the disease.

\section{Materials and methods}

A cohort of 118 school-age children suffering from BA was examined during a period free from attacks at the Pulmo-Allergological Department, Regional Children's Municipal Hospital, Chernivtsi, adhering to the principles of bioethics by means of the simple random sample method. Two clinical groups were formed based on the results of cytomorphological examination of sputum. Group I (main) included 61 schoolchildren $(77.1 \%$ among them were boys) with the signs of the eosinophil phenotype (EP) of asthma detected by the character of bronchial inflammation when eosinophil granulocytes were present in the sputum at a level of $\geq 3 \%$. Group II (comparison) included 57 patients $(52.6 \%$ boys, $\mathrm{P}<0.05$ ) with a lower number of eosinophils in the sputum, and due to this fact the non-eosinophil variant of bronchial inflammatory response was detected in those children, and thus - noneosinophil phenotype (NP) of BA. The value of relative content of eosinophils in the sputum of group I of patients was $13.3 \pm 1.8 \%$, and in group II $-1.94 \pm 0.48 \%(\mathrm{P}<0.001)$, which was indicative of the correct formation of the comparison groups.

The average age of the representatives of groups I and II was $11.9 \pm 0.42$ and $11.04 \pm 0.39$ years respectively $(P>0.05)$. Differences between the groups in the age of first onset of BA were not found; thus in group I asthma made its first onset at the age of $2.2 \pm 0.11$, and in the comparison group this index was $2.1 \pm 0.12(\mathrm{P}>0.05)$.

In group I an intermittent course of eosinophil phenotype of bronchial asthma (EPBA) was registered in 2 cases (3.3\%), mild persistence - in 10 children $(16.4 \%)$, and in group II (the comparison group) - in 5 patients $(8.8 \%)$ and only in 6 patients $(10.5 \%$, $\mathrm{P}>0.05)$ respectively. The atopic form prevailed among the representatives of group II ( $59.6 \%$ against $40.0 \%, \mathrm{P}<0.05)$, and the mixed form was found in patients with EPBA $(60.0 \%$ against $40.4 \%, \mathrm{P}<0.05$ ). The groups were compared by the main clinical signs, and certain evident differences between the groups did not affect the results obtained but only emphasized the phenotype heterogeneity of the disease and the presence of different phenotype variants in sick children.

The control of the disease was evaluated by means of a questionnaire containing clinical-spirometrical findings according to which a value of 10 and lower reflected a controlled course of BA, 11-16 a partially controlled course of the disease, higher than 17 indicated an uncontrolled variant of BA (Boulet et al., 2002). Quantitative and qualitative cytological content of the sediment was detected in sputum of patients, the results were considered to be a group-forming sign: when $>3 \%$ eosinophil granulocytes were found in the sputum the patients were included into group I, patients with a lower number were placed in clinical group II ( the comparison group). At the same time, the fluid fraction above the sediment obtained after sputum was centrifuged and the content in this of human eosinophil cationic protein (ECP) was detected by means of ELISA-method according to the technique worked out by the producer and using reagents Aviscera Bioscience, Inc. (USA). ECP reflected cytotoxic activity of sputum eosinophils, and its average content in the cohort of all the patients was $2.28 \pm 2.2 \mathrm{ng} / \mathrm{ml}$ (from 0 to maximum value $-9.2 \mathrm{ng} / \mathrm{ml}$ ).

The markers of oxygen-dependent metabolism of eosinophil granulocytes in the blood and sputum were studied using the data of the spontaneous and stimulated NBT-test (B. H. Park's method in modification of Klimov et al. (1988), nitroblue tetrazolium test). The reserve of microbicidal ability of these cells was calculated as the difference between the results of the spontaneous and the stimulated NBT-test. Phagocytic function of acidophilic leukocytes in the peripheral blood and sputum was detected by means of E. F. Chernushenko's method.

Interleukin-5 (IL-5) content, which is directly connected with eosinophil functions, was examined in the blood serum by means of the solid-phase immune-enzymatic method using the reagents "IFABest" (RF) according to the recommendations of the producer.

The results obtained were analyzed by means of the computer package Statistica 6.0 (StatSoft Inc., USA) using parametric and non-parametric methods of calculation and by means of clinicalepidemiological analysis.

\section{Results and discussion}

The study demonstrated that in the cohort of the examined patients the eosinophil phenotype of bronchial asthma (EPBA) was found 1.5 times more frequently among the boys, while the noneosinophil phenotype of bronchial asthma (NPBA) was detected more frequently among the girls. Therefore, the male gender was associated with an increased risk of EPBA: odds ratio (OR) index was 7.3 (95\% CI: $1.69-8.23)$, relative risk (RR) - 1.63 (95\% CI: $1.02-2.59)$, attributive risk (AR) -0.3 . Post-test probability $(\mathrm{PP}+)$ of presence of eosinophil variant of bronchitis among boys increased by $16.6 \%$, and among girls (PP-) decreased by $15.1 \%$.

Persistence of BA of moderate severity occurred in every second $(50.9 \%)$ representative of clinical group II, and among the patients with EPBA it was in 1.5 times less $(\mathrm{P}=0.05)$. By contrast, the severe course prevailed significantly among the patients of the clinical group I. The indices of asthma control during initial examination did not differ among the patients with alternative phenotypes of BA, and on completion of the 3rd month of the basic therapy they appeared to be significantly worse among patients with NPBA. Thus, the average score by clinical-instrumental scale to evaluate asthma before the course of treatment in group I was $18.16 \pm 1.03$, and in group II $-18.17 \pm 1.28(\mathrm{P}>0.05)$. At the same time an uncontrolled course of the disease was registered in $55.4 \%$ of the patients in the survey with signs of EPBA, and in group II - in $52.8 \%$ cases $(\mathrm{P}>0.05)$.

There was a tendency to the reduction of the absolute and relative number of eosinophils in the peripheral blood of patients in clinical group I. This may well indicate migration of those effector cells into the "target organ". The average index of relative content of eosinophils in the peripheral blood among the representatives of group I was $5.82 \pm 0.63 \%$, and in children with the signs of NPBA $6.02 \pm 0.74 \%(\mathrm{P}>0.05)$, and average indices in both groups of absolute eosinophil number (AEN) in the blood were $0.37 \pm 0.04$ and $0.41 \pm 0.05$ respectively $(\mathrm{P}>0.05)$. Considering $\mathrm{AEN}$ index 
$\geq 0.5$ as a paraclinical marker of blood eosinophilia, it was found that in group I it was registered in $27.9 \%$ of the patients, and in group II ( the comparison ) - in $35.1 \%(\mathrm{P}>0.05)$. Therefore, the index of AEN $\geq 0.5$ was associated with an inceased risk of NPBA: $\mathrm{OR}=1.4$ (95\% CI: 0.69-3.05), RR $=1.2$ (95\% CI: 0.69-2.02), $\mathrm{AR}=0.08$. At the same time, post-test probability $(\mathrm{PP}+)$ of presence of the non-eosinophil variant of bronchitis increased only by $5.7 \%$, and with lower values of AEN (PP-) it decreased only by $2.6 \%$. Statistically valuable differences in the indices of oxygendependence microbicidal capacity of eosinophils in the peripheral blood and sputum of children with alternative phenotypes of BA were not found (Table 1). Table 1 presents the results of the NBTtest of eosinophil granulocytes (\%) in the blood and sputum in groups I and II of patients. Thus, the lowest reserve of oxygendependent microbicidal capacity of eosinophils in sputum was found in patients with EPBA signs, which was indicative of their leading role in allergic inflammation of the respiratory tract. Negative values of the reserve of NBT-test indices of eosinophils in sputum (as the difference between stimulated and spontaneous results) increased the risk (OR) of EPBA presence by 6.1 times (95\% CI: 0.6557.15), at the same time RR was 1.2 (95\% CI: $0.20-7.47)$, and AR 0.17 . The negative reserve of NBT eosinophils in sputum as the test to verify EPBA showed the following diagnostic values: specificity $-83.3 \%(95 \%$ CI: $35.9-99.9)$, predicted value of a positive result $-95.6 \%$ (95\% CI: 78.1-99.9), spread - 86.9\% (95\% CI: $73.7-95.1)$, accuracy $-58.7 \%$ (95\% CI: 43.2-73.0).

The indices of phagocytic function of eosinophil granulocytes in the peripheral blood and sputum in patients with alternative phenotypes of BA also emphasized the role of these effector cells in allergic inflammation of the respiratory tract (Table 2).

Table 1

Markers of oxygen-dependent metabolism of eosinophils in the blood and sputum in children with eosinophil phenotype of bronchial asthma

\begin{tabular}{lccc}
\hline \multirow{2}{*}{ Indices } & \multicolumn{2}{c}{ NBT spontaneous, \% } & \multicolumn{2}{c}{ NBT stimulated, \% } & blood \\
\cline { 2 - 4 } & blood & sputum & $18.8 \pm 0.97$ \\
Eosinophil phenotype of bronchial asthma & $17.6 \pm 2.13$ & $15.6 \pm 4.58$ & $15.7 \pm 3.86$ \\
Non-eosinophil phenotype of bronchial asthma & $17.3 \pm 1.26$ & $20.2 \pm 6.25$ & $19.2 \pm 1.34$ \\
P & $>0.05$ & $>0.05$ & $>0.05$ \\
\hline
\end{tabular}

Table 2

Indices of phagocytic function of eosinophils in the blood and sputum in children from the clinical groups of comparison

\begin{tabular}{lccc}
\hline \multirow{2}{*}{ Indices } & \multicolumn{2}{c}{ Phagocytic activity, \% } & \multicolumn{2}{c}{ Phagocytic number, standard units } \\
\cline { 2 - 4 } & blood & sputum & blood \\
\hline Eosinophil phenotype of bronchial asthma & $68.7 \pm 6.36$ & $62.1 \pm 2.34$ & $3.24 \pm 0.29$ \\
Non-eosinophil phenotype of bronchial asthma & $73.6 \pm 6.03$ & $26.7 \pm 2.54$ & $3.02 \pm 0.53$ \\
P & $>0.05$ & $<0.05$ & $0.52 \pm 0.53$ \\
\hline
\end{tabular}

The presence of statistically important differences in the markers of phagocytic function of eosinophils in the sputum of children correlated with preliminary suggestions concerning active involvement of these cells into the inflammatory process of the bronchi, which was emphasized by an increased content of IL-5 in the blood serum in the representatives of clinical group I. Thus, IL-5 content in the blood serum of children with EPBA was $5.99 \pm$ $1.74 \mathrm{ng} / \mathrm{ml}$, in patients of group II it was only $1.99 \pm 0.49 \mathrm{ng} / \mathrm{ml}$ $(\mathrm{P}<0.05)$. When the index of IL-5 in the blood was more than $2.0 \mathrm{ng} / \mathrm{ml}$ the risk of EPBA increased: $\mathrm{OR}=2.5$ (95\% CI: 0.49 12.64), $\mathrm{RR}=1.4$ (95\% CI: $0.61-3.34)$, and $\mathrm{AR}-0.2$. The indices of diagnostic value of this paraclinical test in verification of EPBA appeared to be rather modest: sensitivity $-62.5 \%$ (95\% CI: 35.4 84.8 ), specificity $-60.0 \%$ (95\% CI: $26.2-87.8)$, predicted value of a positive result $-71.4 \%$ (95\% CI: 41.9-91.6), spread - 61.5\% (95\% CI: 40.6-79.8), accuracy - 61.5\% (95\% CI: 40.6-79.8). When IL-5 in the blood serum was $>2.0 \mathrm{ng} / \mathrm{ml}$ post-test probability of EPBA increased by $11.0 \%$, and in case of a negative result of the test it was $11.5 \%$ lower.

Eosinophil cationic protein (ECP), detected in the sputum fluid above the sediment as a marker of intensified degranulation of cells in the bronchi proved the presence of mainly eosinophil-mediated inflammation of the respiratory tract of patients with EPBA. Thus, the average content of this cytotoxic mediator in sputum of patients of group I reached $2.72 \pm 0.35 \mathrm{ng} / \mathrm{ml}$, and in the comparison group - only $1.74 \pm 0.34 \mathrm{ng} / \mathrm{ml}(\mathrm{P}<0.05)$. When the content of ECP in sputum was $>1.0 \mathrm{ng} / \mathrm{ml}$ the risk of eosinophil inflammation of the respiratory tract increased significantly: $\mathrm{OR}=4.1(95 \% \mathrm{CI}$ : 1.5-11.2), $\mathrm{RR}=2.0$ (95\% CI: 1.4-3.0), and AR-0.34. This paraclinical index as a marker of EPBA possessed the following diagnostic value: sensitivity - 78.6\% (95\% CI: 63.2-89.7), specificity $-52.9 \%$ (95\% CI: $35.1-70.2)$, spread $-55.3 \%$ (95\% CI: 43.4-66.7), accuracy $-67.1 \%$ (95\% CI: 55.4-77.5). With the above mentioned ECP content in the sputum post-test probability of EPAB presence increased by $12.5 \%$, and in case of a negative result of the test it decreased by $21.2 \%$.

In our opinion, these results facilitate the verification of EPBA as they make it possible to differentiate the choice of medicines for the basic treatment of BA. On completion of the course of antiinflammatory therapy the average score in the questionnaire containing clinical-spirometrical findings in groups I and II was $12.22 \pm 1.34$ and $14.71 \pm 1.38(\mathrm{P}>0.05)$ respectively, although a controlled course was found in $55.6 \%$ of patients with the eosinophil variant and only in $23.8 \%$ of the children with NPBA $(\mathrm{P}<0.05)$. Therefore, the efficacy of the standardized (Order of the Ministry of Public Health of Ukraine № 868, 2013) basic anti-inflammatory therapy in patients of clinical group I was higher in comparison to the children with the signs of NPBA, which was illustrated by the reduced risk of inadequate control of the disease: the index of absolute risk decrease (ARD) was 31.8\%, relative risk (RR) $57.1 \%$ with necessary minimal number of patients (NMNP) -1.75 .

We have shown that application of markers of inflammatory response of the body such as eosinophils in the blood and sputum, indices NBT-test blood granulocytes, intracellular and extracellular content of cationic proteins in patients with asthma is highly sensitive to the nature of inflammation in the airways, which coincides with the literature (Wilson et al., 2000; Shirai et al., 2006; Broide, 2008; Johansson et al., 2008). For instance, we found that a severe course had a high probability of prevailing in patients with the eosinophilic phenotype of asthma compared to patients with neutrophilic inflammation of the airways. However, the literature mentions the severe course of bronchial asthma as being due to the presence of neutrophil inflammation of the bronchi (Gibson et al., 1999; Mann et al., 2006), although data on the nature of the relationship inflammation of the bronchi to the severity of the disease remain controversial (Li et al., 2003; Kikuchi et al., 2005). In particular, according to the literature, an elevated relative content of eosinophilic granulocytes in sputum of patients with eosinophilmediated type of disease is considered a marker of efficacy of antiinflammatory therapy, which was confirmed in our study, given the increase in the number of patients with a controlled disease course after a course of basic anti-inflammatory therapy. The effectiveness of standardized basic anti-inflammatory therapy in patients with the eosinophilic phenotype of asthma was significantly higher compared with children who showed signs of the neutrophil phenotype, emphasizing the idea that the increase in the number of neutrophils 
in the airways was registered in late phase allergic reactions in patients with so-called "fateful" and "nocturnal asthma", as well as severe forms of asthma (Wenzel, 2003; Kamath et al., 2005; Haldar et al., 2007).

\section{Conclusions}

The chances of developing eosinophil phenotype of asthma are greater in the following cases: with males (7.3 times), negative reserve of NBT-test of eosinophils in the sputum (6.1 times), IL-5 content in the blood serum over $2.0 \mathrm{ng} / \mathrm{ml}$ (by 2.5 times) and eosinophil cationic protein content in the sputum over $1.0 \mathrm{ng} / \mathrm{ml}$ (by 4.1 times). The highest specificity in verifying eosinophil inflammation of the bronchi occurred with the negative reserve of the NBT-test of eosinophils in the sputum (83.3\%), and the highest sensitivity in its detection - the content of ECP over $1.0 \mathrm{ng} / \mathrm{ml}$ (78.6\%). Consideration of the character of a local inflammatory process in the bronchi enhances the efficacy of the basic therapy of bronchial asthma by $57.1 \%$ with its eosinophil phenotype less than in every second patient.

\section{References}

Beasley, R., Roche, W. R., Roberts, J. A., \& Holgate, S. T. (1989). Cellular events in the bronchi in mild asthma and after bronchial provocation. American Review of Respiratory Disease, 139(3), 806-817.

Boulet, L-P., Boulet, V., \& Milot, J. (2002). How should we quantify asthma control?: A proposal. Chest, 122(6), 2217-2223.

Broide, D. H. (2008). Immunologic and inflammatory mechanisms that drive asthma progression to remodeling. Journal of. Allergy and Clinical Immunology, 121, 560-570.

Chatila, T. A., Li, N., Garcia-Lioret, M., Kim, H. J., \& Nel, A. E. (2008). T-cell effector pathway in allergic diseases: Transcriptional mechanisms and therapeutic targets. Journal of Allergy and Clinical Immunology, $121,812-823$.

Gibson, P. G., Norzila, M. Z., \& Fakes, K. (1999). Pattern of airway inflammation and its determinants in children with acute severe asthma. Pediatric Pulmonology, 4, 261-270.

Global Initiative for Asthma. Global Strategy for asthma management and prevention, 2016.

Haldar, P., \& Pavord, I. D. (2007). Noneosinophilic asthma: A distinct clinical and pathologic phenotype. Journal of Allergy and Clinical Immunology, 119(5), 1043-1052.

Jacobsen, E. A., Lee, N. A., \& Lee, J. J. (2014). Re-defining the unique roles for eosinophils in allergic respiratory inflammation. Clinical and Experimental Allergy, 44(9), 1119-1136.
Johansson, M. W., Kell, E. A., Busse, W. W., Jarjour, N. N., \& Mosher, D. F. (2008). Up-regulation and activation of eosinophil integrins in blood and airway after segmental lung antigen challenge. The Journal of Immunology, 180, 7622-7635.

Kamath, A. V., Pavord, I. D., Ruparelia, P. R., \& Chilvers, E. R. (2005). Is the neutrophil the key effector cell in severe asthma? Thorax, 60, 529-530.

Kato, M., Suzuki, M., Hayashi, Y., \& Kimura, H. (2006). Role of eosinophils and their clinical significance in allergic inflammation. Expert Review of Clinical Immunology, 2(1), 121-133.

Kikuchi, S., Nagata, M., \& Kikuchi, I. (2005). Association between neutrophilic and eosinophilic inflammation in patients with severe persistent asthma. International Archives of Allergy and Immunology, 137(Suppl. 1), 7-11.

Li, A. M., Lex, C., Zacharasiewicz, A., \& Wong, E. (2003). Cough frequency in children with asthma: Correlation with lung function, exhaled nitric oxide, and sputum eosinophil count. Thorax, 58, 974-978.

Litvinova, L. S., Kolobovnikova, Y. V., Knutareva, E. N., Grigoryeva, E. S., Suvorova, E. V., Novitskiy, V. V., \& Riazantseva, N. V. (2006). Cytotoxic potential of eosinophilic granulocytes in patients with eosinophilic syndrome. Newsletter of Siberian Medicine, 5(3), 26-30.

Madison, J. M., \& Schramm, C. M. (2000). Cationic proteins and bronchial hyperresponsiveness. American Journal of Respiratory Cell and Molecular Biology, 22(5), 513-516.

Mann, B. S., \& Chung, K. F. (2006). Blood neutrophil activation markers in severe asthma: Lack of inhibition by prednisolone therapy. Respiratory Research, 7, 59.

Motojima, S., Frigas, E., Loegering, D. A., \& Gleich, G. J. (1989). Toxicity of eosinophil cationic proteins for guinea pig tracheal epithelium in vitro. American Review of Respiratory Disease, 139(3), 801-805.

Novitskiy, V. V., Riazantseva, N. V., Litvinova, L. S., Kolobovnikova, Y. V., Grigoryeva, E. S., \& Suvorova, E. V. (2006). The mechanisms of disorders of eosinophils and immunocytes in the formation of gross blood eosinophilia. Newsletter of Siberian Medicine, 2, 32-34.

Pavord, I. D., 2013. Eosinophilic phenotypes of airway disease. ATS Journal, 10, 143-149.

Ponikau, J. U., Winter, L. A., Kephart, G. M., Squillace, D. L., \& Hershcovitch, M. D. (2015). An immunologic test for chronic rhinosinusitis based on free intranasal eosinophilic major basic protein. International Forum of Allergy \& Rhinology, 5(1), 28-35.

Shirai, T., Inui, N., \& Chida, K. (2006). Correlation between peripheral blood T-cell profiles and airway inflammation in atopic asthma. Journal of Allergy and Clinical Immunology, 118, 622-626.

Wenzel, S., 2003. Mechanism of severe asthma. Clinical and Experimental Allergy, 33, 1622-1628.

Wilson, N. M., Bridge, P., Spanevello, A., \& Silverman, M. (2000). Induced sputum in children: Feasibility, repeatability, and relation of findings to asthma severity. Thorax, 55, 768-774. 